\title{
Optical Analysis of Germanium Carbide Thin Films Deposited by Reactive Pulsed Laser Ablation
}

\author{
A. Mahmood ${ }^{1}$, M. Iqbal ${ }^{1,2}$, Zahid Ali ${ }^{1}$, H. Z. Shafi ${ }^{1}$, A. Shah ${ }^{1}$, D. Batani ${ }^{2}$ \\ ${ }^{1}$ National Institute of Laser and Optronics, Nilore, Islamabad, Pakistan \\ Phone: 9251-9290231, Ext: 3206, Fax: 9251-2208051 \\ Email: muniqbal@yahoo.com \\ ${ }^{2}$ Dipartimento di Fisica, "G.Occhialini”, University of Milano-Bicocca, \\ Piazza della Scienza 3, 20126 Milan, Italy. \\ E-mail:batani@mib.infn.it
}

\begin{abstract}
We deposited germanium carbide (GeC) thin films by Pulsed Laser Deposition (PLD) technique. Plasma plume created in PLD makes it possible to grow $\mathrm{GeC}$ thin film at room temperature. Thickness and optical constants were measured using spectroscopic ellipsometer (SE) in UltravioletVisible range. Relationship between optical constants with methane gas pressure was analyzed. Xray Photoelectron Spectroscopy (XPS) was used for compositional analyses. It is observed that higher pressure of methane gas for the growth of $\mathrm{GeC}$ by PLD is responsible for carbon agglomeration at the surface of the film. Moreover, the tunability of the band gap was observed from 1.6 to 2.8 $\mathrm{eV}$ with the pressure of methane gas.
\end{abstract}

DOI:10.2961/jlmn.2010.03.0004

Key Words: reactive pulsed laser deposition, ellipsometry, X-ray Photoelectron Spectroscopy, atomic force microscopy

\section{Introduction}

There is worldwide interest in Pulsed Laser Deposition (PLD) for the growth of high quality thin films. PLD, in principle is very simple technique where laser pulses are used to remove the material from the target. The vaporized material from the target consists of neutral atoms, ions and electrons and is generally termed as laser produced plasma plume. This transient, highly luminescence plasma plume expands rapidly from the target surface. The velocities of the plasma particles have a profound effect on the quality of the film as it is easier for atoms to occupy the required sites because of higher mobility of atoms, which results in lesser number of defects [1]. It is reported that the transfer of complex stoichiometry is possible by PLD technique [2]. Moreover, the presence of ions and their higher kinetic energies make it possible to have reactive deposition at low temperature.

$\mathrm{GeC}$ carbide is wide band gap semiconductor material with attractive electronic and optoelectronic properties is a promising candidate for photovoltaic and other electrooptic applications since the addition of $\mathrm{C}$ to Ge reduces the lattice dimensions enough to allow an ordered growth on silicon substrate, while it increases the band gap to be closer to that of c-Si rather than c-Ge [3-4]. GeC has been deposited with different techniques such as hollow cathode sputtering [5], molecular beam epitaxy [6], and magnetron sputtering [7]. In the present work reactive PLD technique is used to grow $\mathrm{GeC}$ thin films. The aim of this work is to investigate the effect of gas $\left(\mathrm{CH}_{4}\right)$ pressure on the quality of the films prepared by reactive PLD technique.

\section{Experimental set-up}

Si substrates (100) were cleaned before deposition ultrasonically by the standard method. Target of pure $\mathrm{Ge}$ $(99.999 \%)$ was ablated by using excimer-Krypton Fluoride $(\mathrm{KrF})$ pulsed laser $(\lambda=248 \mathrm{~nm})$ in the presence of methane as the reactive gas. Methane gas (99.99\%) was used as the source of carbon in the film(s). Samples were deposited by varying the pressure of methane from 0 to 75 $\mathrm{mT}$ (with increment of 25 milliTorr (mT) for each sample). Laser energy was $200 \mathrm{~mJ}$ with repetition rate of $5 \mathrm{~Hz}$. Number of pulses used for each film was 6000 . Base pressure was set at $10^{-9}$ Torr. Deposition time for each sample was 20 minutes.

Number of physical characteristics such as band gap, light absorption and its color can be determined using complex refractive index as function of photon energy. Ellipsometery provides a convenient way to measure the refractive index and thickness of thin films. For the optical analyses of $\mathrm{GeC}$ thin films the "SE-850 Ellipsometer", by SENTECH instruments $\mathrm{GmbH}$, was employed.

Ellipsometer measures the change in polarization expressed as $\Psi$ and $\Delta$. For the measurement of film thickness and optical constants it is necessary to perform model dependent analyses of the ellipsometric data. The directly measurable parameters $\Psi$ and $\Delta$ are related to film properties according to the relation:

$$
\rho=\frac{r_{s}}{r_{p}}=(\tan \psi) e^{i \Delta}
$$

Where, $r_{p}$ and $r_{s}$ are Fresnel reflection coefficients for the parallel and perpendicular components of polarized light 
respectively for curve fitting, we used the Cauchy model to simulate the refractive index wavelength dependency, which is

$$
\begin{aligned}
& n(\lambda)=n_{0}+C_{0} \frac{n_{1}}{\lambda^{2}}+C 1 \frac{n_{2}}{\lambda^{4}}+\ldots \\
& k(\lambda)=k_{0}+C_{0} \frac{k_{1}}{\lambda^{2}}+C 1 \frac{k_{2}}{\lambda^{4}}+\ldots
\end{aligned}
$$

Where $\mathrm{C}_{0}$ and $\mathrm{C}_{1}$ are constants to avoid large number of values of $\mathrm{n}_{1}, \mathrm{k}_{1}, \mathrm{n}_{2}, \mathrm{k}_{2}, \ldots$ and independent of material while $k_{0}$ and $k_{1}$ etc will depend upon material [8].

In order to investigate the surface morphology of the deposited films Atomic force microscopy was carried out. The experimental facility was provided by the "Nano Materials Lab", DCME, and PIEAS.

\section{Results}

Fig. 1 shows the XPS results of the deposited films. The composition of the samples prepared at $0,25,50$ and 75 $\mathrm{mT}$ is shown in table 1 .

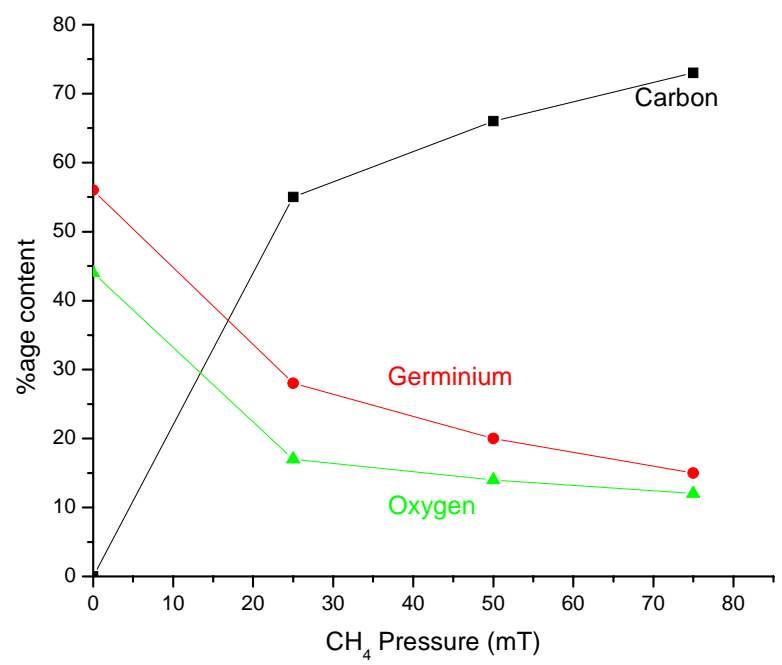

Figure 1: XPS results for GeC samples

\begin{tabular}{|c|c|c|c|c|}
\hline $\begin{array}{c}\text { S } \\
\#\end{array}$ & $\begin{array}{c}\mathbf{C H}_{\mathbf{4}} \\
\text { Pressure } \\
(\mathbf{m T})\end{array}$ & $\begin{array}{c}\text { \%age } \\
\text { Oxygen } \\
\text { content }\end{array}$ & $\begin{array}{c}\text { \%age } \\
\text { Carbon } \\
\text { content }\end{array}$ & $\begin{array}{c}\text { \%age Ger- } \\
\text { manium con- } \\
\text { tent }\end{array}$ \\
\hline 01 & 0 & 44 & 0 & 56 \\
\hline 02 & 25 & 17 & 55 & 28 \\
\hline 03 & 50 & 14 & 66 & 20 \\
\hline 04 & 75 & 12 & 73 & 15 \\
\hline
\end{tabular}

Table 1: XPS results of GeC samples.

These results show that the carbon concentration increases as the pressure of the methane gas is increased. It is $0 \%$ for the sample prepared at $0 \mathrm{mT}$ and $55 \%, 66 \%$ and $75 \%$ for the samples prepared at $25 \mathrm{mT}, 50 \mathrm{mT}$ and $75 \mathrm{mT}$ respectively. It is also observed from the XPS results that the oxygen content in the samples decreases as we increase the pressure of methane gas. It is $44 \%, 17 \%, 14 \%$ and $12 \%$ for the sample prepared at $0 \mathrm{mT}, 25 \mathrm{mT}, 50 \mathrm{mT}$ and $75 \mathrm{mT}$ respectively.

For the analysis of the prepared films by Spectroscopic Ellipsometery, the spectra for ellipsometric angles $\Psi$ and $\Delta$ were recorded. Using a multilayer model, the best possible fitting was achieved. The multilayer stacking was consisted of a composite surface layer of $\mathrm{GeO}_{2}$ (3-15 nm thick), a layer of $\mathrm{GeC}+$ Voids (88-143 nm thick), a layer of $\mathrm{SiO}_{2}(0$ $15 \mathrm{~nm}$ thick) and the $\mathrm{Si}(100)$ substrate as shown in figure 2.

\begin{tabular}{|c|}
\hline $\mathrm{GeO}_{2}$ \\
\hline $\mathrm{GeC}+$ Voids \\
\hline $\mathrm{SiO}_{2}$ \\
\hline $\mathrm{Si}(100)$ \\
\hline
\end{tabular}

\begin{tabular}{|c|c|c|c|c|}
\hline $\begin{array}{l}\mathbf{S} \\
\#\end{array}$ & $\begin{array}{c}\mathrm{CH}_{4} \\
\text { Pressure } \\
(\mathrm{mT})\end{array}$ & Material & $\begin{array}{c}\text { Thickness } \\
\text { (nm) }\end{array}$ & voids \%age \\
\hline \multirow{3}{*}{01} & \multirow{3}{*}{0} & $\mathrm{GeO}_{2}$ & 30.09 & \multirow{3}{*}{-} \\
\hline & & $\begin{array}{l}\mathrm{GeC}+ \\
\text { Voids }\end{array}$ & 0 & \\
\hline & & $\mathrm{SiO}_{2}$ & 0 & \\
\hline \multirow{3}{*}{02} & \multirow{3}{*}{25} & $\mathrm{GeO}_{2}$ & 11.36 & \multirow{3}{*}{41} \\
\hline & & $\begin{array}{l}\text { GeC + } \\
\text { Voids }\end{array}$ & 133.10 & \\
\hline & & $\mathrm{SiO}_{2}$ & 14.59 & \\
\hline \multirow{3}{*}{03} & \multirow{3}{*}{50} & $\mathrm{GeO}_{2}$ & 15.21 & \multirow{3}{*}{36} \\
\hline & & $\begin{array}{l}\mathrm{GeC}+ \\
\text { Voids }\end{array}$ & 88.40 & \\
\hline & & $\mathrm{SiO}_{2}$ & 9.04 & \\
\hline \multirow{3}{*}{04} & \multirow{3}{*}{75} & $\mathrm{GeO}_{2}$ & 3.75 & \multirow{3}{*}{20} \\
\hline & & $\begin{array}{l}\mathrm{GeC}+ \\
\text { Voids }\end{array}$ & 142.02 & \\
\hline & & $\mathrm{SiO}_{2}$ & 0 & \\
\hline
\end{tabular}

Figure 2: Multilayer model for ellipsometric measurements.

Table 2: Ellipsometric results for GeC samples

The fraction of the voids was considered to be 20-41\% (see Tab. 2 in detail). The fitting parameters are $\mathrm{N}_{0}=2.128$, $\mathrm{N}_{1}=2355.6, \mathrm{~N}_{2}=-2399.2, \mathrm{~K}_{0}=0.151, \mathrm{~K}_{1}=-603.203$ and $\mathrm{K}_{2}=1976.131$. The Maxwell garnet effective medium approximation theory was used to calculate the refractive index of the composite layer according to the relation given below.

$$
\frac{n_{e}^{2}-n_{h}^{2}}{n_{e}^{2}+2 n_{h}^{2}}=f \frac{n_{1}^{2}-n_{h}^{2}}{n_{1}^{2}+n_{h}^{2}}
$$


Where $n_{e}, n_{h}$ and $n_{1}$ are the complex refractive indices for the effective medium, host medium and the inclusion respectively.

Nearly a perfect agreement between the modeled and experimental spectra was obtained as shown in figure 3 .

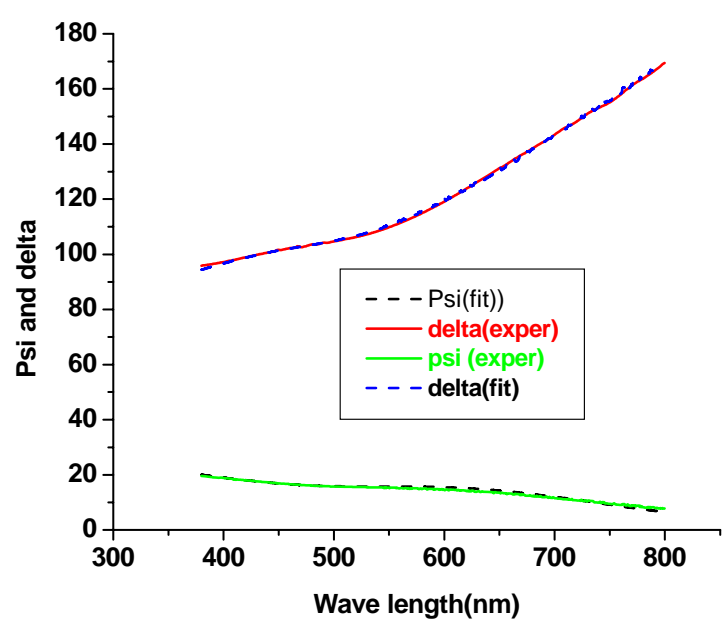

Figure 3: Experimental and fitted results of $\Psi$ and $\Delta$

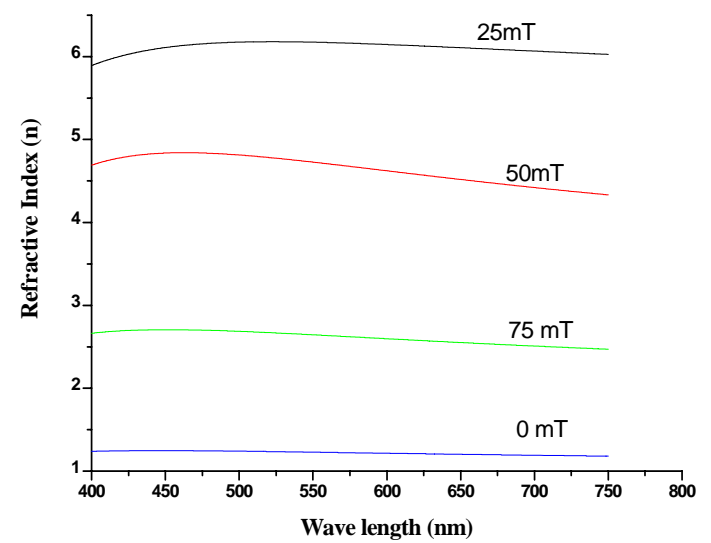

Figure 4: Refractive index vs, wavelength at different $\mathrm{CH}_{4}$ pressures

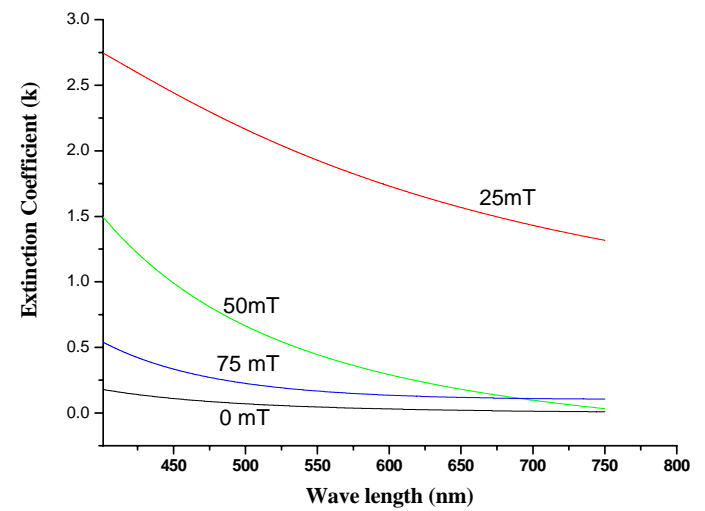

Figure 5: Extinction coefficient Vs wavelength.

Figures 4 and 5 show the real and imaginary parts of the refractive index as a function of the wavelength for the thin films prepared under the indicated deposition conditions. Refractive index tends to be decreasing over the whole wavelength range for the samples prepared at $25 \mathrm{mT}, 50 \mathrm{mT}$ and $75 \mathrm{mT}$. Similar kind of behavior is also observed for extinction coefficient. Figures 6 and 7 show variation of refractive index and extinction coefficient with the pressure of methane gas. In order to calculate the absorption coefficient $(\alpha)$ of the deposited films, the following relation was used [9].

$$
\alpha=\frac{4 \pi K}{\lambda}
$$

Where $\pi \approx 3.14$ and $\mathrm{K}$ is extinction coefficient. The band gap of the deposited films was obtained by plotting square of the product of absorption coefficient and photon energy verses photon energy as shown in Figure 8. Results are shown in figure 9.

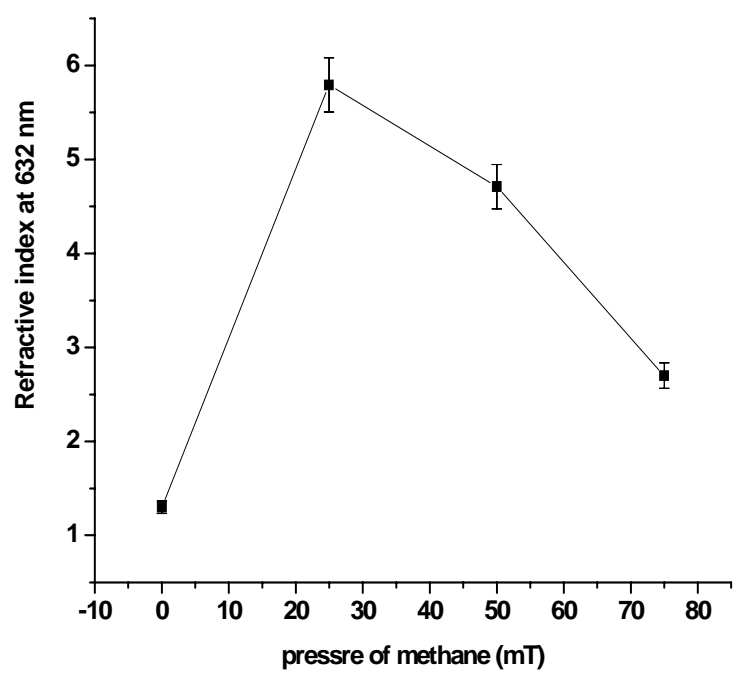

Figure 6: Refractive index Vs Pressure of methane gas

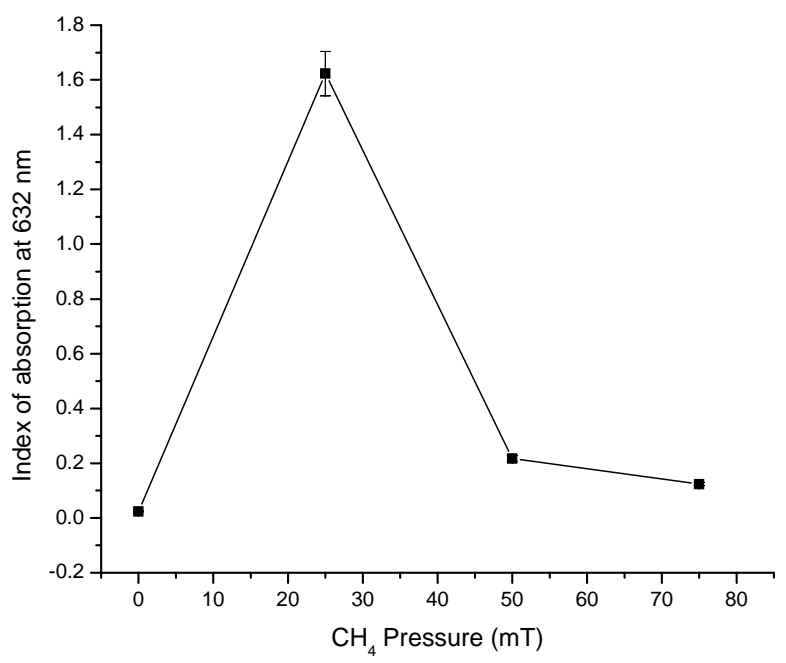

Figure 7: Index of absorption Vs Pressure of methane gas 


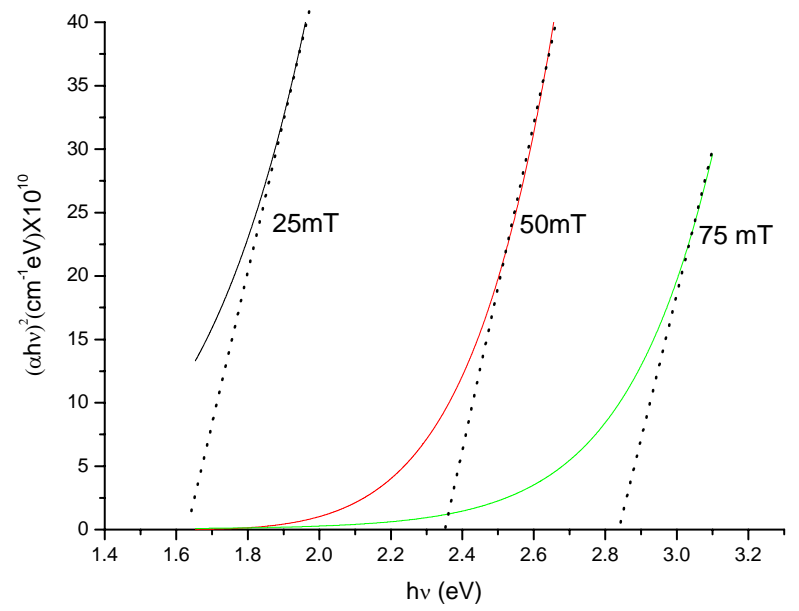

Figure 8: Square of the product of absorption coefficient and Photon energy Vs Photon energy

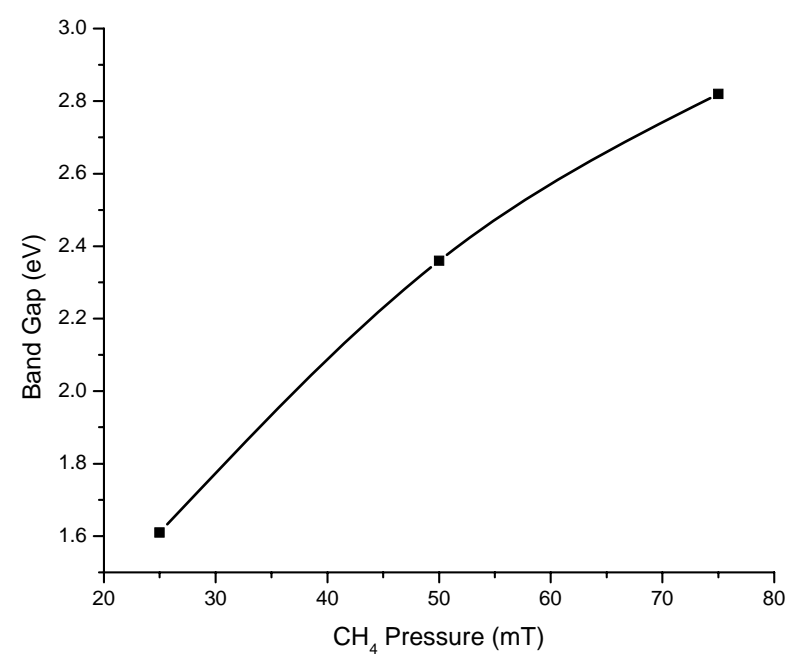

Figure 9: Band gap Vs Methane pressure

Figure 10 shows the AFM images of the samples deposited at different deposition conditions. Fig 10(a) shows three-dimensional view of the uncoated Si (100) sample. The area scanned for each sample was $2.5 \mu \mathrm{m} \times$ $2.5 \mu \mathrm{m}$. Average height along z-axis (perpendicular to the surface of deposited films) was found to be $26.43 \mathrm{~nm}$. Fig 10 (b) shows three-dimensional view of the sample deposited at gas pressure of $25 \mathrm{mT}$. The average height of cluster/island along z-axis in this case was found to be 45.02 $\mathrm{nm}$. Fig 10(c) shows the surface morphology of the sample deposited at a gas pressure of $50 \mathrm{mT}$. The average height of the particulate/island grown was observed to be 40.40 . Fig 10 (d) shows the micrograph of the sample deposited at a gas pressure of $75 \mathrm{mT}$. The average height of the particulate/island grown was observed to be $55.05 \mathrm{~nm}$. Much larger size of the islands is obvious from this micrograph.
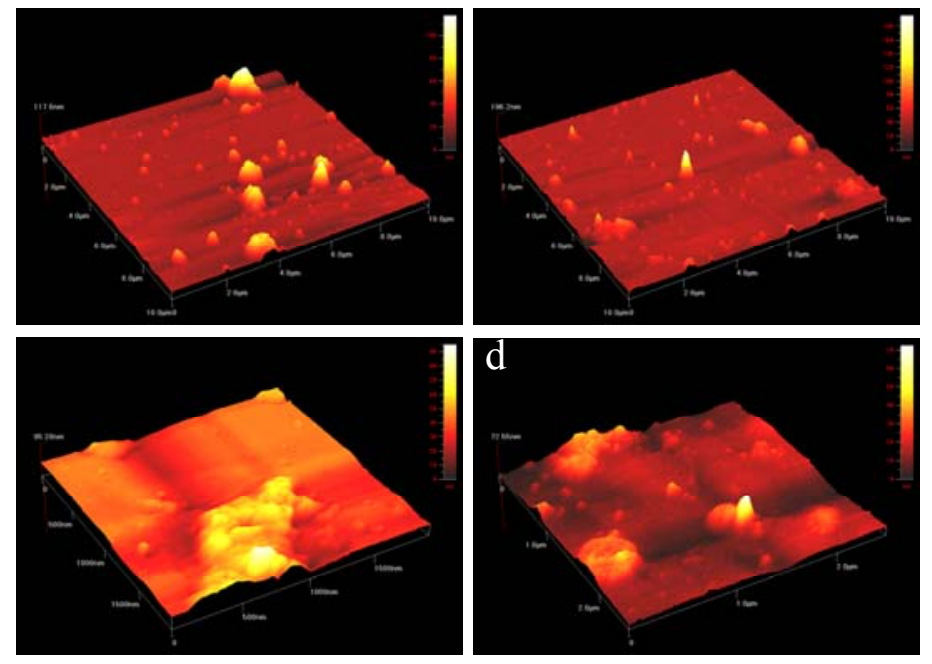

Figure 10: AFM images of the deposited films

\section{Discussions}

Deposition parameters have a profound effect on the composition, optical and surface properties of the films. The ablated germanium has the dominant part of ionic germanium because of $\mathrm{KrF}$ laser $(\lambda=248 \mathrm{~nm})$ that is more reactive which provides the possibility to form $\mathrm{GeC}$. From the XPS results it is observed that the carbon concentration in the deposited films increases as we increase the pressure of the methane gas. This is quite understandable that as the pressure of the methane gas is increased, it increases the amount of carbon available for the deposition. XPS results also depict that there is oxygen content in the deposited films and it decreases as the carbon content in the film increase. Decrease in oxygen content in the film might be attributed carbon increment. As the carbon concentration increases it reacts with germanium to form $\mathrm{GeC}$, which is less vulnerable for oxide formation. Furthermore the increase in carbon concentration decrease the Ge content on the surface, hence less Ge is available for the formation of $\mathrm{GeO}_{2}$.

Results of the Spectroscopic Ellipsometery showed that the refractive index decreases as the pressure of the methane gas increases. We observed that the refractive index of the films prepared at $25 \mathrm{mT}$ is closer to that of $\mathrm{Ge}$ and it decrease with pressure of methane gas to a value closer to that for carbon for the film prepared at $75 \mathrm{mT}$. This is in good agreement with the XPS results as it depicts that by increasing the $\mathrm{CH}_{4}$ pressure carbon concentration is increasing. For the sample prepared at $0 \mathrm{mT}$ has a very small value of refractive index. This reveals that the film prepared at $0 \mathrm{mT}$ is mostly of $\mathrm{GeO}_{2}$. Smaller value of refractive index for this film may also be due to film thickness which is only $\sim 30 \mathrm{~nm}$. Pressure of methane gas has also a strong influence on the extinction coefficient of the films. At pressure of $25 \mathrm{mT}$ or above, it decreases with the increase in pressure while for the sample prepared at $0 \mathrm{mT}$ it has smallest value. Moreover the band gap of the films deposited at higher $\mathrm{CH}_{4}$ pressure is greater than that of the samples prepared at low $\mathrm{CH}_{4}$ pressure except for the sample prepared at $0 \mathrm{mT}$ where it is found out to be $\sim 3 \mathrm{eV}$. This increase in band gap is again attributed to carbon concentration as it is suggested that the increase in carbon con- 
tent is followed by the consistent increase of $\mathrm{sp}^{3} \mathrm{Ge}-\mathrm{C}$ bonds. The band gap of the sample prepared at $0 \mathrm{mT}$ is quite high than other samples because of the formation of $\mathrm{GeO}_{2}$ as observed from the XPS and ellipsometric results.

Ellipsometric results show that there are voids in the deposited films and fraction of voids tends to be decreasing with the increase in pressure of methane gas. This shows that the density of the film also improves with the increase in carbon content. The decrease in voids fraction might be correlated to the change in the surface free energy, enhancement of surface diffusion kinetics or a combination of both.

AFM results reveal that as $\mathrm{CH}_{4}$ pressure is increased the surface of the deposited film become rougher. In general, gas pressure and plasma plume in laser ablation cause the non-uniformity in the films. In our experiments roughness seems to be increasing with the pressure of the methane gas and we can say that by increasing the pressure, deposited films become non-uniform. We have also observed from our XPS and ellipsometric results that the film prepared at $75 \mathrm{mT}$ has excessive carbon which is $\sim 73 \%$ more. This excessive amount of carbon causes carbon agglomeration on the surface of the film. This carbon agglomeration can also contribute towards non-uniformity of the films.

\section{Conclusions:}

Analysis of SE, XPS and AFM data indicate that the pressure of the methane gas has a profound effect on the composition, surface morphology and on the optical constants of films deposited by reactive pulsed laser ablation technique. Carbon content in the films increases with the increase in the pressure of methane gas. We conclude from our results that higher pressure of methane gas not only deteriorates the surface morphology but also the stoichiometry of the films as well. For high quality thin films by PLD, an optimized value of reactive gas pressure should be employed. It is observed that increase in carbon content, increases the band gap, so it is possible to tune band gap of $\mathrm{GeC}$ by controlling the concentration of carbon.

\section{References}

1. M.P. Chuchman, A. K. Shuaibov, Emission characteristics and properties of laser-plume Germanium plasma, Plasma Physics Reports 34 (2008) 306-311.

2. R .W. Mathew, R. Shinar, A. P. Constant, Reactive pulsed laser deposition of micro crystalline Ge-based thin films, Proc. Mat. Res. Soc. 762 (2003) 655-660.

3. M. Kumru, A Comparison of the optical, IR, electron spin resonance and conductivity properties of a-Ge $\mathrm{Ge}_{1}$ ${ }_{\mathrm{x}} \mathrm{C}_{\mathrm{x}}: \mathrm{H}$ with a-Ge:H and a-Ge thin films prepared by R.F sputtering, Thin Solid Films 198 (1991) 75-84.

4. J. Shinar, H.S. Wu, R. Shinar , H.R. Shanks, An IR, optical, and electron-spin-resonance study of asdeposited and annealed $a-\mathrm{Ge}_{1-x} \mathrm{C}_{x} \mathrm{H}$ prepared by $\mathrm{rf}$ sputtering in $\mathrm{Ar} / \mathrm{H}_{2} / \mathrm{C}_{3} \mathrm{H}_{8}$, J. Appl. Phys. 62 (1987) 808812.

5. J. L. Huguenin-Love, R.J. Soukup, N.J. Ianno, J.S. Schrader, D.W. Thompson, V.L. Dalal, Optical and crystallographic analysis of thin films of $\mathrm{GeC}$ deposited using a unique hollow cathode sputtering tech- nique, Materials Science in Semiconductor Processing 9 (2006) 759-763.

6. M. Krishnamurthy, B. K. Yang, W. H. Weber, Microstructural development and optical properties of epitaxial $\mathrm{Ge}_{1-\mathrm{x}} \mathrm{C}_{\mathrm{x}}$ alloys on Si (100), Appl. Phys. Lett. 69 (1996) 2572-2574.

7. C. Q. Hu, W. T. Zheng, J. J. Li, Q. Jiang, H. W. Tian, X. Y. Lu, J. W. Liu, L. Xu, J. B. Wang, . $\mathrm{Ge}_{1-\mathrm{x}} \mathrm{C}_{\mathrm{x}}$ double-layer antireflection and protection coatings, Applied Surface Science 252 (2006) 8135-8138.3

8. Software Manual. SpetraRay advanced fit module. Germany: SENTECH instrument GmbH (1999).

9. H. Fujiwara, Spectroscopic elipsometery principles and applications, John Wiley \& Sons, England, (2007).

(Received: March 23 , 2010, Accepted: August 17, 2010) 